\title{
A Novel Processing Methodology for Traffic-Speed Road Surveys Using Point Lasers
}

\author{
Wenda $\mathrm{Li}^{\circledR}$, Michael Burrow, Nicole Metje, Yueyue Tao, and Gurmel Ghataora
}

\begin{abstract}
The rapidly increasing traffic volumes using local road networks allied to the implications of climate change drive the demand for cost-effective, reliable and accurate road condition assessment. A particular concern for local road asset managers is the loss of material from the road surface known as fretting which unchecked can lead to potholes. In order to assess the road condition quantitatively and affordably, a system should be designed with low complexity, be capable of operating in a variety of weather conditions and operate at normal trafficspeeds. Many different techniques have been developed for road condition assessment such as ground penetrating radar, visual sensors and mobile scanning lasers. In this work, the use of the point laser technique for scanning the road surface is investigated. It has the advantages of being sufficiently accurate, is relatively unaffected by levels of illumination and it produces relatively low volumes of data. In this work, road fretting/surface disintegration was determined using a novel signal processing approach which considers a number of features of reflected laser signals. The proposed methodology was demonstrated using data collected from the UK's local road network. The experimental results indicate that the proposed system can assess road fretting to an accuracy which is comparable to a visual inspection, and at Information Quality Level (IQL) 3 which is sufficient for tactical road asset management whereby road sections requiring treatment are selected and appropriate treatments identified.
\end{abstract}

Index Terms-Road condition assessment, point laser, signal processing, peak detection.

\section{INTRODUCTION}

$\mathbf{T}$ HERE is a strong relationship between road condition and road user costs (i.e. travel time, fuel consumption, vehicle maintenance and safety) [1]. However, the condition of a road deteriorates over time due to the combined effects of the traffic and environmental impacts. Timely and appropriate preventative road maintenance is therefore required to ensure road user costs are kept within acceptable limits. This necessitates the periodic assessment of road condition. Surfaced urban and rural roads (i.e. local roads), which typically constitute over $90 \%$ of a country's road network, are a major challenge. The extent of the local road network lends itself to automated condition assessment which is much faster, more accurate

Manuscript received April 15, 2019; revised October 15, 2019; accepted November 18, 2019. This work was supported by the U.K. Technology Strategy Board through the Innovate U.K., under Project 91429-554255 and Grant 103891. The Associate Editor for this article was D. F. Wolf. (Corresponding author: Wenda Li.)

The authors are with the Department of Civil Engineering, University of Birmingham, Birmingham B15 2TT, U.K. (e-mail: w.li@bham.ac.uk; m.p.burrow@bham.ac.uk; n.metje@bham.ac.uk; g.s.ghataora@bham.ac.uk; yxt643@student.bham.ac.uk).

Digital Object Identifier 10.1109/TITS.2019.2957238 and cost-effective than manual approaches. Together with intelligent signal processing, a fast computing unit and a digital storage device, automated assessment has the potential to deliver a quantitative analysis of road condition at traffic speeds in real-time [2]. For the assessment of surfaced local roads, automated systems need to be capable of assessing the predominant deterioration types of local roads, namely cracking and fretting (road surface disintegration which leads to the formation of potholes). Their early detection is vital to enable appropriate preventative maintenance. However, because of the variety of surface types used to construct local roads and the difficulty in assessing cracking and fretting using automated means, their evaluation is usually carried out by trained inspectors walking along the road or by using windshield surveys [3]. The latter involves an observer sitting inside a moving vehicle.

Vehicle-based systems have been used widely to capture road surface information as they are fast, accurate and cost effective [4] when compared to other approaches such as airborne and satellite systems. The development of vehicle-based systems has been driven by advances in direct geo-referencing, sensor technologies and computer processing algorithms. This work focus on the measurement of road fretting. Fretting is caused by the loss of aggregate particles in the road surface due to the aging of the binder and the subsequent loss of adhesion between the binder and aggregates. Fretting unchecked, leads to the development of potholes, a major issue for the safety of road traffic [5], and the deterioration of the road structure.

The Highways Agency Road Research Information System 2 (HARRIS2) data collection vehicle has been developed to assess the condition of the UK's strategic road network and has a number of downward facing point lasers as part of its scanning system [6]. In this paper, a robust signal processing approach is presented that identifies areas of road surface fretting from the data obtained from the HARRIS2 point laser system. This data can be regarded as being a combination of information concerning the road texture and road fretting and unwanted noise associated with the sensor and dynamic motion of the vehicle. The first steps in the signal processing approach are to remove the sensor noise and the effects of the dynamic motion of the vehicle. Then the road texture and fretting components of the data are treated as a peak detection problem which is addressed using an automatic estimation process. Afterwards, outputs from each point laser are combined using an empirical approach. The experimental 
results show that, with the proposed concepts, a point laser system that scans the road surface in the longitudinal direction is comparable to visual inspection.

Compared to previous work presented in the literature [6] [10], the proposed point laser approach contributes the following:

- A system which can assess road fretting in real time using a low complexity signal processing algorithm which requires relatively low volumes of data.

- A novel peak detection algorithm to estimate road texture.

- A system which is verified using survey data collected at normal traffic speeds from a real environment. The results indicate that the proposed approach can provide comparable results to a visual survey.

The paper is organized as follows: Section II briefly reviews the literature; Section III describes the system and the datasets used in this paper; Section IV presents the signal processing algorithms; Section $\mathrm{V}$ provides the detection performance and analysis; conclusions are in Section VI.

\section{RELATED WORK}

\section{A. Road Surface Sensors}

A variety of sensor technologies and associated processing techniques have been proposed for road surface condition assessment, however efficient solutions remain in the early stage of development. These technologies can be categorized as: 1) mechanical wave, 2) electromagnetic wave (EM) and 3) image based techniques.

In the first category, ultrasound [11], [12] and acoustic [13], [14] based approaches have been used for the assessment of buried infrastructure. These approaches have been shown to be capable of accurately measuring the thickness of buried objects and the associated hardware is relatively inexpensive and can be mounted on a moving vehicle or a trailer [13]. However, they are unable to detect fine cracks and small areas of fretting and water on the road surface can also significantly reduce their detection performance [11], [14]. These limitations therefore restrict the application potential of ultrasound systems for the assessment of local road conditions.

Electromagnetic (EM) wave techniques, including Ground Penetrating Radar (GPR) and laser-based systems, are also used to assess road condition. GPR systems have shown some promise when successfully used to detect highly cracked areas of road surface, assess airfield pavement condition and determine the integrity of buried infrastructure among other things [15], [16]. Limitations of GPR systems for road surface condition inspection concern their inability to operate accurately when there is water on the road surface and their resolution which is not sufficient to detect minor cracks and small areas of fretting on the road surface. Laser systems are a well-developed technology, and are becoming increasingly popular for many commercial applications as they lack many of the disadvantages of GPR, ultrasonic and visual systems [4], [9], [17]. Their advantages include the ability to operate in most environmental conditions and a high resolution and frequency of data capture. An early approach described by [7] presents the use of mobile scanning lasers to measure road surface cracking automatically. The system records the road surface in three dimensions and extracts longitudinal profile data to evaluate possible defects. Work by [18] demonstrates the use of a Mobile Laser Scanning (MLS) system for road marking classification. A more advanced MLS system is presented in [4] that can extract road surface features, including road markings and certain types of road surface cracks. The systems described by [4], [7], [18] all generate a large amount of data and require complex algorithms to process the data. Consequently, these systems are unable to process data in real-time and as a result they need offline processing which necessitates significant digital storage, increasing the hardware costs of data collection and processing. Moreover, the large amount of energy emitted by the MLS system poses a danger to nearby flora and fauna.

Visual systems can provide sufficient resolution to recognize theoretically even the smallest cracks and are used commercially for road condition inspection [19]-[21]. Work by [20] proposes a percolation model to identify road cracks from images of the road surface with high level of noise. A Deep Convolutional Neural Network (DCNN) was developed by [19] to extract road surface cracks from photographs taken by a smart phone. Images of the road surface can also be obtained using Light Detection and Ranging (LiDAR) techniques and these could potentially be analysed to assess road condition. For example, research by [22] presents the use of LiDAR to detect the edges of a road to assist autonomous vehicle navigation. The angle of the sun during data collection however has been shown to affect significantly the performance of LiDAR systems [23].

\section{B. Studies on Point Laser Systems}

Unlike the MLS system, the point laser system does not scan the entire road surface but only the longitudinal profile. This significantly reduces both the volume of data to be processed and the computational complexity of the associated signal processing. A disadvantage of a single point laser is that it cannot provide transverse information and therefore an array of point lasers is required to obtain transverse information. A study by [8] developed a system consisting of a point laser and an algorithm which scrutinizes histograms of the relative distance of the road surface from a datum to identify areas of fretting on the road surface. Work by [6] further expanded this approach and proposed an algorithm to identify fretting based on the Root Mean Square (RMS) value of the measured changes in road surface height with respect to a given datum. A similar study has also been presented in [10] which measured road surface texture depth. i.e. the depth between physically repeating features on the road surface due to aggregate particles present within the road surface. However, the work by [6], [8], [10] are constrained in that the systems described are only suitable for road surfaces with similar texture, whereas in practice the road texture encountered during a survey varies considerably due to the different road construction types apparent within a typical local road network. As a result, the above approaches require information concerning the road type to be specified manually. This reduces their degree of automation and potentially 
their accuracy since it is not straightforward for the operator of a data collection vehicle to visually distinguish between road surface types when driving at traffic speeds. Besides, the dynamic motions from the vehicle can reduce significantly the performance of texture based methods. Such vehicle motions can result in up to $100 \mathrm{~mm}$ of vertical displacement which are much larger than the depth of fretting which is usually between $0.5 \mathrm{~mm}$ and $2 \mathrm{~mm} \mathrm{[10]}$.

\section{Studies on Time-Series Data}

Point laser data captured from a moving vehicle is analogous to time-series data since the frequency at which the laser system takes a measurement of the road surface is a function of the vehicle's speed and the frequency of the laserbased measurement system. For analysing time series data, the peak detection method has been shown to be a powerful approach in the fields of electrocardiography (ECG) [24], human activity classification and location detection [25] and accelerometer based transportation mode recognition [26]. Similarly, the Change Point Detection (CPD) technique has been applied successfully to a broad range of real-world applications. For example, monitoring the change in behaviour in a smart house [27], predicting changes in climate [28], the analysis of human activity [29] and speech recognition [30]. Time-series data can also be analysed by supervised and unsupervised learning approaches [27]. For supervised learning, the division between two data points can be used as an indicator of possible change points [31], or classifiers (such as decision trees) can be used to generate interpretable rules [32]. For unsupervised learning, the distance between data points can be used as a divergence measure [27], Bayesian approaches have been used to estimate changes in probability distributions [30] and the Kernel-based method has been used to map data points into higher-dimensional space [33]. The choice of an appropriate algorithm depends on the structure of the data point, the inherent noise and the purpose of the application.

\section{SySTEM AND DATASET}

\section{A. HARRIS2 System}

The HARRIS2 system used in this study has been designed for the automated assessment of the UK's strategic road network [7]. The equipment installed on the vehicle includes: 1) a central system with an Inertial Measurement Unit (IMU), a GPS unit and a Distance Measurement Unit (DMU); 2) five ProfiCura 2D point lasers [34] for longitudinal road scanning; 3) a MLS system [35] for three-dimensional road scanning and; 4) other sensors including LiDAR and a forward facing video camera. All the above components are integrated on a van (Ford TDCi). Fig. 1 shows the five point lasers mounted in the front of the vehicle. Each laser is aligned at 90 degrees to the road surface and the lasers are distributed horizontally at an equal spacing. The lasers scan the longitudinal profile of the road surface. Data from each point laser are synchronized and processed by the central system.

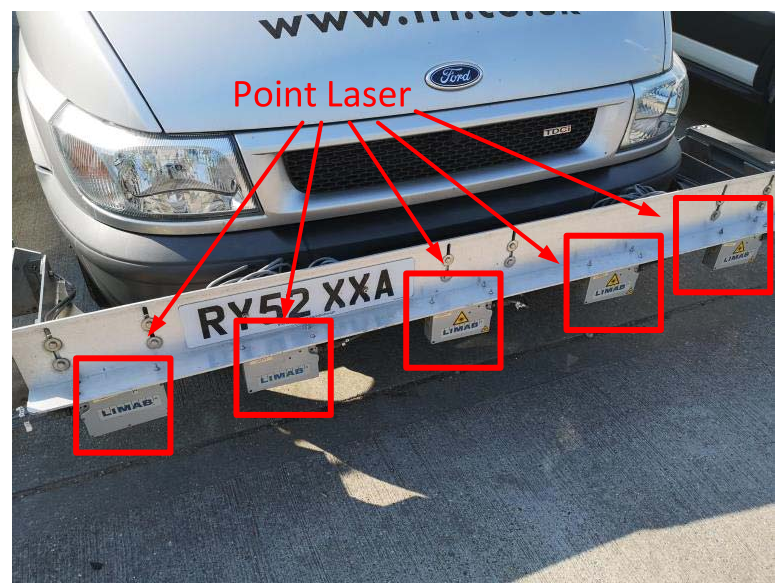

Fig. 1. The point laser system mounted on the HARRIS2.

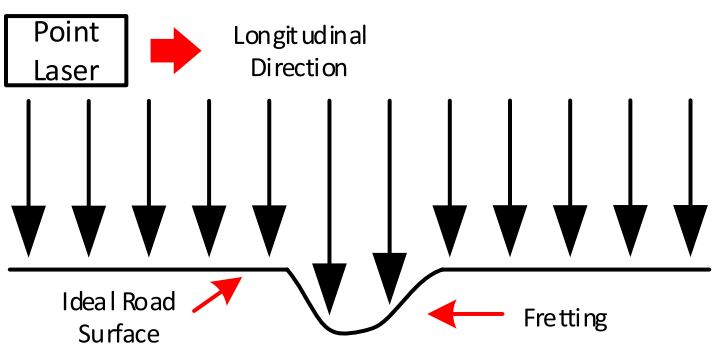

Fig. 2. A schematic of idealized fretting detection using a point laser.

\section{B. Working Principles of a Point Laser}

A schematic of an idealized point laser system detecting an area of fretting is shown in Fig. 2. The figure illustrates a point laser traversing the longitudinal direction of the road with an area of fretting in the middle of the idealised section of road. The point laser transmits a laser beam towards the ground and measures the Time-of-Flight (ToF) of the reflected beam at a particular frequency. In HARRIS2, the frequency of the laser beam transmission is controlled by the vehicle's speed so that a constant longitudinal resolution is maintained, i.e. same amount of data is collected over a $20 \mathrm{~m}$ section of road irrespective of the vehicle speed. An ideal smooth intact road surface should reflect the light from the laser homogeneously to the laser sensor as indicated in Fig. 2. In contrast, in an area of fretting, the light has a number of reflection paths back to the point lasers sensor. Consequently, a number of irregular measurements are recorded compared to the measurement of the intact road surface. In practice, the measurement of fretting is further complicated as the road surface is not smooth, but is textured due to the presence of aggregate particles. The approach proposed in this research to measuring fretting under these circumstances is explained further in Section IV C.

Three parameters are crucial to the performance of laser based systems in detecting fretting. These are the longitudinal resolution $R_{L}$ (i.e. the distance along the road between successive laser measurements), the transverse resolution $R_{T}$ (i.e. the horizontal spacing of the lasers) and the vertical resolution $R_{V}$ (i.e. the minimum size of object that can be measured in the 
(a)
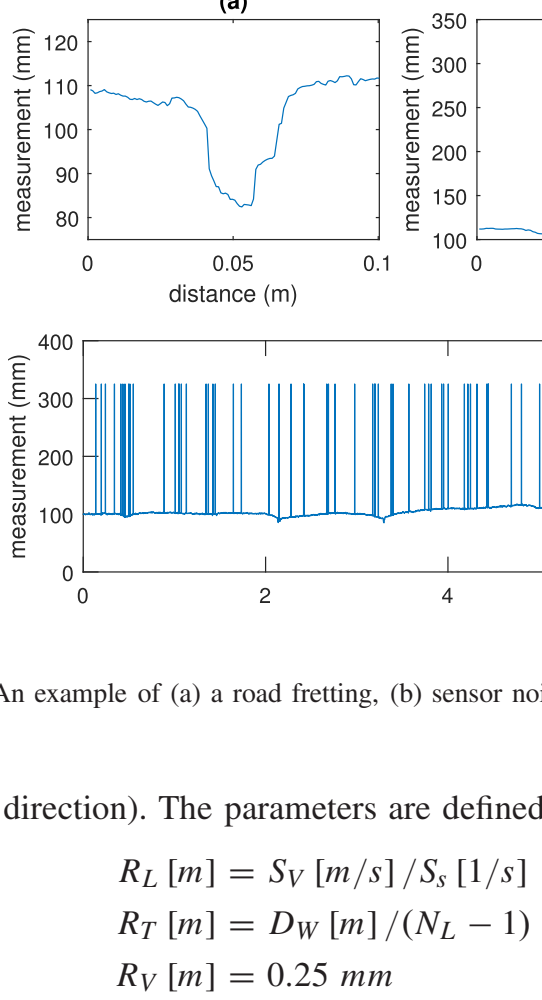

(b)

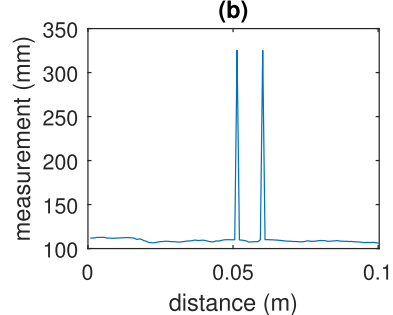

$$
\begin{aligned}
& R_{T}[m]=D_{W}[m] /\left(N_{L}-1\right) \\
& R_{V}[m]=0.25 \mathrm{~mm}
\end{aligned}
$$

(c)

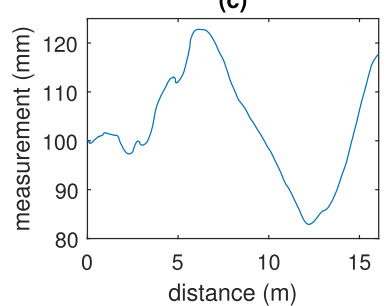

(d)

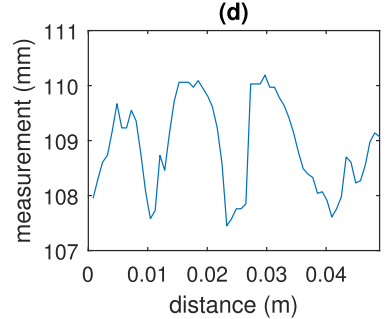

(e)

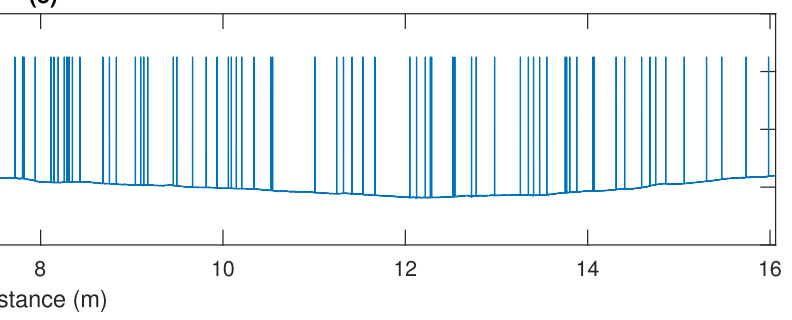

Fig. 3. An example of (a) a road fretting, (b) sensor noise, (c) vehicle dynamic motions (d) road texture and (e) the raw data from single point laser.

vertical direction). The parameters are defined as follows:

where $S_{V}$ is the vehicle speed, $S_{S}$ is the scanning rate, $D_{W}$ is the scanning width (transverse direction) of the system (4.2 metre in this work), $N_{L}$ is the total number of point lasers (five in the case of HARRIS2), and $R_{V}$ is $0.25 \mathrm{~mm}$ for the point lasers fitted on HARRIS2 [34]. From Equation 1, it can be seen that $R_{L}$ is proportional to $S_{V}$ and is inversely proportional to $S_{s}$. $S_{S}$ is controlled by the central unit thus taking into account the vehicle's speed $S_{V}$ to ensure that $R_{L}$ is constant during a road survey. For the roads assessed in this work the speed limit was $80 \mathrm{~km} / \mathrm{h}$ (i.e. $S_{V}=80 \mathrm{~km} / \mathrm{h}$ ) and $R_{L}$ was fixed at $0.803 \mathrm{~mm}$ to achieve a sufficient resolution for micro-texture measurement. Since the number of point lasers is five, using this value in Equation 2, it can be seen that $R_{T}=1.05 \mathrm{~m}$ which apparently is too coarse for the detection of fretting. As a result, the transverse information has not been used in this work.

Three main types of interference may affect the measurement of fretting and they therefore need to be considered by the signal processing algorithms. These are:

- Point laser errors: The main source of laser related errors involve the internal clock in the sensor giving an incorrect measurement of the ToF between the outward and inward laser pulses, the angular resolution of the laser angle encoder and the uncertainty of the beam divergence.

- Vehicle dynamic errors: The vehicle when moving is subject to dynamic vertical motions which can be far larger in magnitude than the depth of road surface fretting. Since the point laser sensor measures the distance from the vehicle to the road surface, the change in distance caused by the occurrence of fretting may be difficult to distinguish from the larger in magnitude change caused by the dynamic motion of the vehicle. This can be regarded as a baseline time series data problem and can be rectified by using an appropriate filter as suggested by [36].

- Texture type: All roads have a textured surface resulting from the materials used in their construction. Surface texture helps to increase road safety by increasing skid resistance and facilitating the removal of water from the road surface. The presence of road surface texture however complicates the measurement of fretting since it introduces variations in the height of the road surface. This issue is further exacerbated as local road networks are constructed with a variety of surface materials depending on a number of factors. These include the speed and volume and type of vehicular traffic using the road, the available finance to build and maintain the road and environmental considerations such as noise reduction [37]. This means that during a survey, the distance to the road surface measured by a laser varies along the surface of a road built from a single type of material and also as a result of different types of road surfaces, irrespective of the presence of fretting.

An example of raw data from a single point laser is provided in Fig. 3(e). The data clearly shows the variation in the height of the road surface. Randomly distributed spikes and troughs are also apparent. This data can be regarded as a combination of four components: (1) fretting (shown in Fig. 3(a)); (2) sensor errors (shown Fig. 3(b)); (3) vehicle dynamic motions (shown in Fig. 3(c)), and (4) road texture (shown in Fig. 3(d)). The magnitude of data point $y(t)$ at time $t$ from a single point laser can be modelled as a superposition of these components as follows:

$$
y(t)=y^{F}(t)+y^{S}(t)+y^{V}(t)+y^{T}(t)+n(t)
$$

where $y^{F}(t)$ is the fretting component at time $t, y^{S}(t)$ is the sensor noise, $y^{V}(t)$ is the component due to the instantaneous vehicle dynamic motions, $y^{T}(t)$ is the component due to texture, and $n(t)$ is the noise from other sources. To determine the amount of fretting, components other than $y^{S}(t)$ need to be removed. The corresponding signal processing to achieve this is presented in Section IV. 


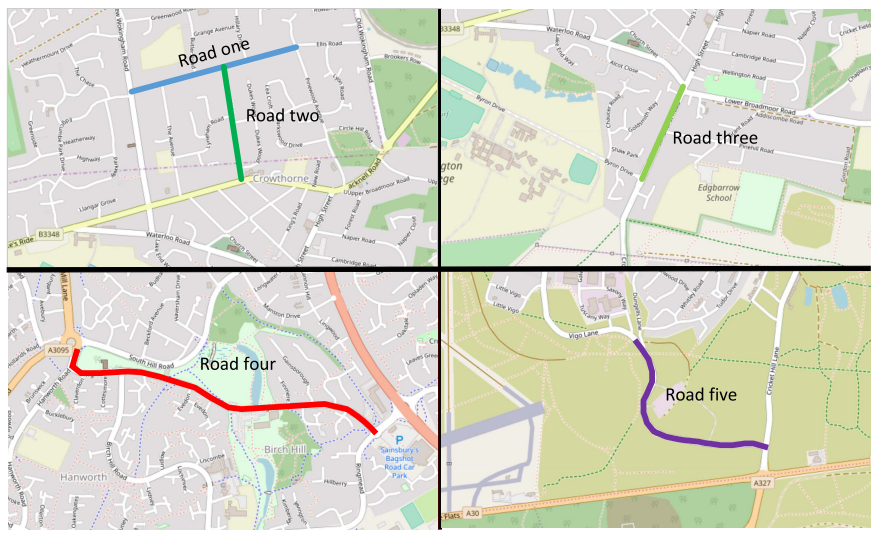

Fig. 4. Survey routine.

TABLE I

SUMMARIZE OF DATASET

\begin{tabular}{|l|l|l|l|}
\hline Road & Description & Length $(\mathrm{km})$ & Direction \\
\hline One & Wokingham Rd to Pinewood Ave & 0.711 & East \\
\hline Two & Duke's ride to Ellis Rd & 0.480 & North \\
\hline Three & Byron Dr to Church St & 0.421 & North \\
\hline Four & Hanworth Rd to Ringmead Rd & 1.337 & East \\
\hline Five & Cricket Hill Lane to Dungells In & 0.831 & West \\
\hline
\end{tabular}

\section{Dataset}

The survey data was collected on local roads near Crowthorne, United Kingdom (longitude $0^{\circ} 47^{\prime} 31.88^{\prime \prime}$ E, latitude $51^{\circ} 22^{\prime} 12.97^{\prime \prime} \mathrm{N}$ ) using the HARRIS2 vehicle on February 22018 (dry surface). Five roads of three different classification were surveyed. These included an A Road (the highest class of classified road in the UK), a B Road (second tier in the UK's classified local road system) and Secondary Road (roads that are unnumbered roads or unclassified roads) (see Fig. 4 and Table I). The roads serve local communities (e.g. town high streets, residential areas) and tourists, and act as through routes for freight traffic. Excessive vehicle loading, changeable weather and a lack of maintenance has resulted in a number of areas of fretting along several of the surveyed roads.

During the road survey, HARRIS2 travelled at speeds ranging between 0 and $80 \mathrm{~km} / \mathrm{h}$, depending on the amount of traffic and the status of traffic signals. The survey started when the driver pressed a start button in the central unit and stopped automatically according to a predefined location. The collected data were then stored and time-stamped. To evaluate the overall detection performance of the proposed methodology visual data were also collected by experienced operators using a windshield survey.

\section{Signal Processing for Point Laser}

This section outlines the signal processing approach developed to assess the amount of fretting. An overview of the proposed methodology of signal processing is shown in Fig. 5. The methodology consists of two main tasks, namely pre-processing and fretting estimation. First, a thresholding method was used to identify and replace the spikes in the data

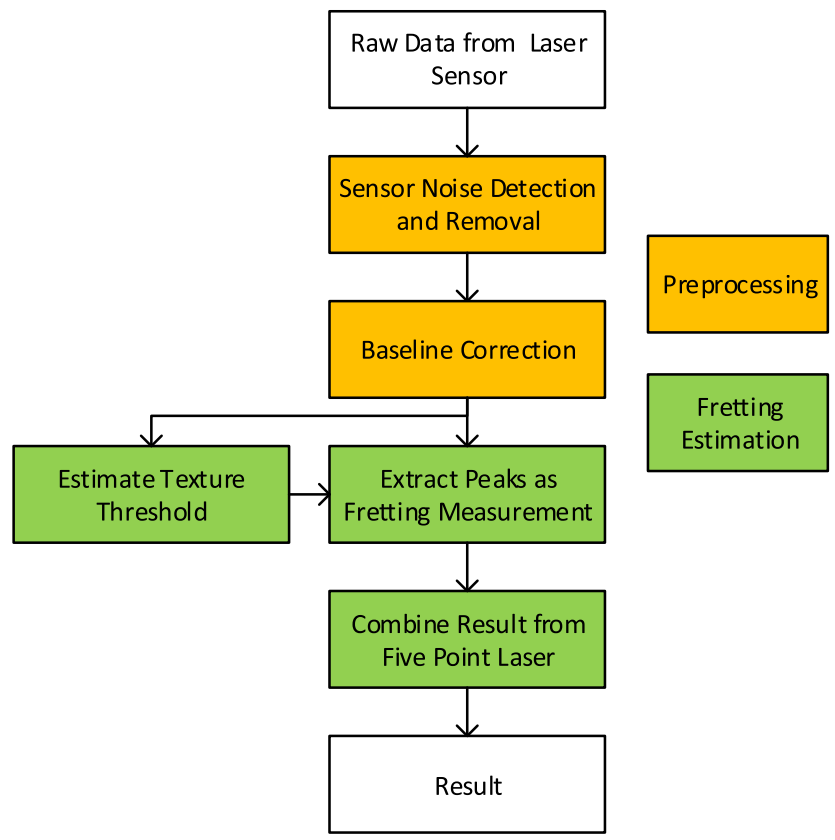

Fig. 5. Signal processing of the proposed point laser system.

that are due to errors associated with the point laser. Thereafter, the Moving Average of Minima (MAM) [38] method was applied to remove the component of the signal due to the dynamic motions of the HARRIS2 vehicle. Thirdly, the road texture and road fretting were analysed using a peak detection approach.

\section{A. Sensor Noise Removal}

As described in Section III, systematic errors from the laser sensor are one of the major noise sources. By analysing the entire dataset, the average error in terms of the number of misclassified data points was approximately $0.5 \%$. Considering that the point laser has a longitudinal resolution of $0.803 \mathrm{~mm}$ this means approximately six errors per metre. If not removed, these erroneous data points could significantly impact the accuracy of fretting detection. An example of the original data points over a 50m section of a road is presented in Fig. 6(a). A large number of saturated measurements are evident over the whole of the $50 \mathrm{~m}$ section. These measurements mask the areas of fretting. It can also be observed that the amplitude of the sensor errors varies unpredictably.

A potential solution is to apply a smoothing filter to suppress the erroneous data points, for example using the commonly used Savitzky Golay (SG) filter. This type of filter replaces a data point by a linear extrapolation of the nearby data points [39]. However, in this data set the data points associated with sensor errors are much higher in value compared to other data points. This is unlike the typical additive white Gaussian noise. Therefore, although it is easy to apply a smoothing filter, the smoothing process would result in a loss of data related to areas of fretting. Another possible solution is to remove the data points that exceed a given threshold value. However, this would cause an imbalance of the data points as the spikes are not distributed evenly across the data set. 

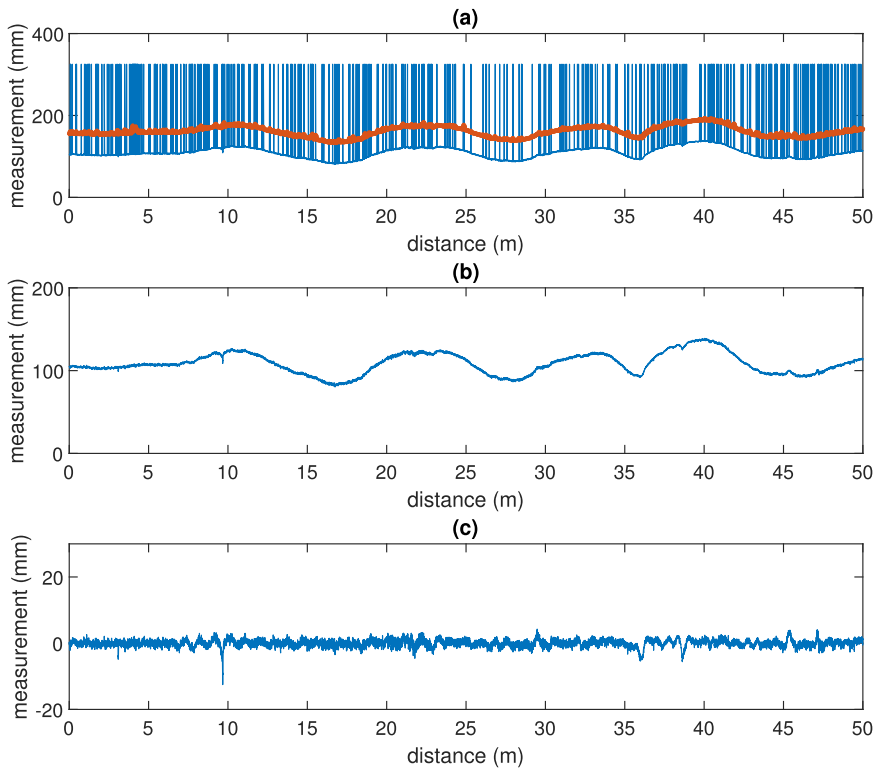

Fig. 6. An demonstration of (a) original data points from one point laser, (b) corresponding cleaned data points and (c) with baseline correction.

From the system aspect, the imbalance of data would further increase the complexity of the implementation process and affect the time-series based signal processing.

Due to the above reasons, a two-step algorithm was developed to deal with the erroneous data points whilst not affecting the other authentic data points. Firstly, a threshold is generated to identify the outlying data points. A single threshold value would not be appropriate as the varying effect of the vehicle dynamic motions could result in authentic data points exceeding the threshold. Consequently, the moving averaging method was used to produce an adaptive threshold. The adaptive thresholding $T(t)$ is calculated as:

$$
T(t)=\sum_{i=-\frac{1}{2} l_{s}}^{\frac{1}{2} l_{s}} c_{i} y(t+i)+w
$$

where $l_{s}$ is the window length (one metre in this work), $c_{i}=1 /\left(l_{s}+1\right)$ is a weighting factor, and $w$ is a constant. For the $50 \mathrm{~m}$ long example dataset, the threshold calculated using Equation 5 is shown in Fig. 6(a) (the thick line). All data points above this threshold are considered to be erroneous.

The second step is to replace the erroneous data with estimated values. For each erroneous data point $y_{i}^{S}$ is replaced by the mean value of its adjacent data points. The mean value is defined as: $y_{i}^{S}=\frac{1}{2 n}\left(y_{i-n}+\ldots+y_{i+n}\right)$, where all data points from $y_{i-n}$ to $y_{i+n}$ are authentic measurements. For the $50 \mathrm{~m}$ long example dataset, the resulting cleaned data points are shown in Fig. 6(b). As can be seen, all the erroneous data have been correctly detected and replaced without any loss in the number of data points.

\section{B. Baseline Correction}

As mentioned above, the dynamic motions of the vehicle can have a significant impact on the measurement of road surface defects. These motions can result in changes in magnitude of the value of an authentic data point of more than $40 \mathrm{~mm}$ as shown in Fig. 6(b). In addition, the duration and frequency of the vehicle dynamic motions are not constant but vary as a function of the number of factors including the vehicle speed and the road surface condition.

In this research, the vehicle's dynamic motion is considered as a baseline correction problem since its effect on the overall signal is to introduce components which have significantly greater fluctuations in magnitude and longer wavelengths compared to those associated with both road texture and fretting. There are many approaches to baseline correction, for example, linear interpolation, monotone minimum, Continues Wavelet Transformation (CWT) and MAM [24]. In this work, MAM was chosen for baseline correction due to its low complexity and relatively small required computational effort. It has also been shown to perform well in dealing with longer wavelength components [24]. Furthermore, the MAM algorithm is similar to the sensor noise removal described in Section IV-A. The first step of the MAM algorithm is to estimate the baseline $\dot{y}^{V}(t)$ by using a moving-average window of the form given by in Equation 5, but without the $w$ constant. A value of $5 \mathrm{~m}$ for the filter length was chosen as it was found to provide a balance between minimizing the effects of vehicle dynamics and not removing too much information. Accordingly the resulting corrected signal, $\hat{y}(t)$, is calculated by Equation 6:

$$
\hat{y}(t)=y(t)-\dot{y}^{V}(t)
$$

The corresponding result of applying the above processes to the $50 \mathrm{~m}$ example dataset is shown in Fig. 6(c). As can be seen, most of the variation due to the dynamic motion of the vehicle has been successfully removed and clearly identifiable area of fretting can be observed at a distance of $10 \mathrm{~m}$ and $38 \mathrm{~m}$. The dynamic motion of a data collection vehicle varies depending on its velocity, mass, suspension system and tyre pressure amongst other things. These motions are of much greater magnitude and wavelength compared to the road texture and fretting even for a low speed or under braking.

\section{Fretting Detection}

Following the removal of the sensor noise and baseline correction, the remaining data points can be considered to be a superposition of road texture $y^{T}(t)$ and road fretting $y^{F}(t)$. As mentioned above road surface texture can vary between roads of different construction. For traffic speed surveys it is impractical for the operator to specify changes in road texture type. Furthermore, the surface texture can vary along a road of the same construction type due to a variety of factors as mentioned above. Therefore, an algorithm which assesses fretting needs to account automatically for variations in road surface type. Consider the example of the laser signal from two roads with different textures in Fig. 7, neither road contains fretting. As can be seen, the depth of texture in Fig. 7(a) is much greater in magnitude when compared to that in Fig. 7(b). The measurements in Fig. 7(a) do not represent an area of fretting but might be considered so if they are occurred within the data shown in Fig. 7(b) as the texture 
(a)
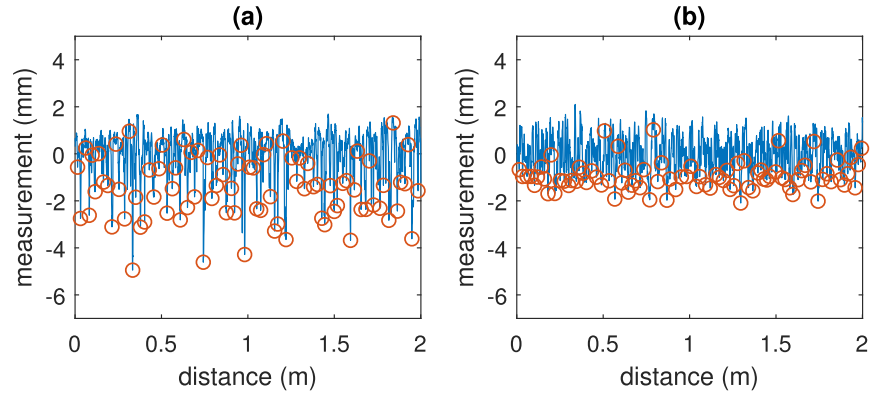

Fig. 7. An example of two types of texture measurement, road texture and detected peaks (circles).

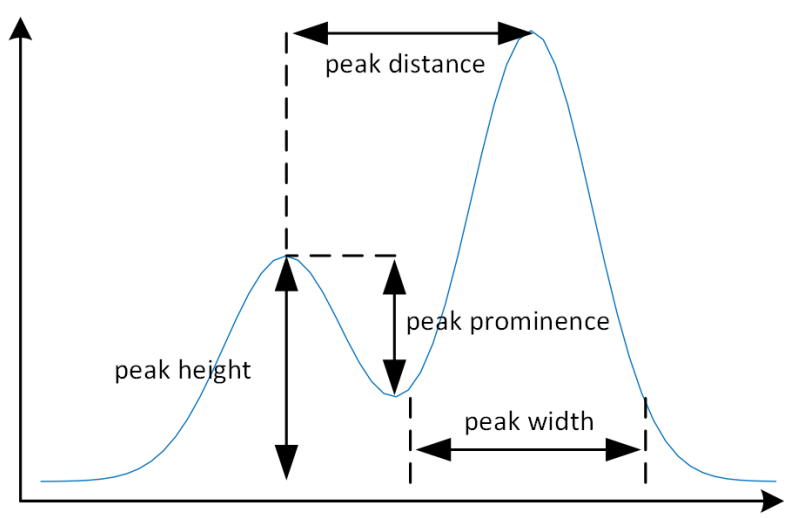

Fig. 8. Feature selection for peak representative.

depth is of greater magnitude. Consequently, it is not possible to identify accurately fretting using a single threshold value based on the expected depth of fretting. Note that, since the fretting is below the road surface, all data points are processed UPSIDE-DOWN.

To aid the detection of fretting, a feature vector is proposed consisting of the peak height, peak width, peak distance (between two adjacent peaks) and peak prominence of perturbations in the road surface as shown schematically in Fig. 8 . Peak height $P_{h}$ measures the distance between the top (crest) of a peak to its trough. Intuitively one might expect a large value of peak height with respect to the rest of the road surface to indicate the presence of fretting. Peak width $P_{w}$ is the width between two reference lines of a peak. Peak distance $P_{d}$ is the distance between two adjacent peaks. It might be expected that the different texture of two roads constructed of different materials will be indicated by dissimilar average peak distances. Peak prominence $P_{p}$ is a measure of the difference between the peak height and its location relative to other peaks. $P_{p}$ is used to distinguish a peak with high value in $\mathrm{Ph}$ but is a member of a group of other large peaks. For convenience, a peak can be written as a feature vector in the form of $\lambda=\left(P_{h}, P_{w}, P_{d}, P_{p}\right)$ and group of peaks as $\lambda=\left[\lambda_{1}, \lambda_{2}, \ldots \lambda_{k}\right]$, where $\lambda$ includes the peaks due to both texture $y^{T}$ and fretting $y^{F}$. Thus, the focus in next step is to automatically identify the $\lambda_{i}$ that belongs to $y^{F}$.

By calculating the feature vector $\lambda_{T}$ for road texture $y^{T}$, it is possible to estimate the type of road texture and to use this to derive a classification which can be used to distinguish
TABLE II

Feature Vectors for Two Types of RoAd Texture

\begin{tabular}{|l|l|l|}
\hline texture & (a) $(\mathrm{mm})$ & (b) $(\mathrm{mm})$ \\
\hline Peak height $\left(P_{h}\right)$ & 2.43 & 1.87 \\
\hline Peak width $\left(P_{w}\right)$ & 3.27 & 2.33 \\
\hline Peak distance $\left(P_{d}\right)$ & 5.08 & 4.61 \\
\hline Peak prominence $\left(P_{p}\right)$ & 0.64 & 0.61 \\
\hline
\end{tabular}

between fretting and texture. For example, consider Table II which shows the calculated average feature values of the circled peaks shown in Fig. 8. As can be seen, the feature vector resulting from texture (a) is very different compared to that from texture (b). By inspection, the feature vector of texture (a) has significantly higher values of peak height, width and distance. These differences could potentially therefore be used to classify road texture.

Identifying areas of fretting can, from the above, be considered as a process of identifying those peaks which contain irregular $\lambda$ values when compared to $\lambda_{T}$. Based on the UK standard for identifying fretting [3], four rules were developed to help fretting detection as follows:

1) Texture depth is the distance between the top of an aggregate and the binding material in which it sits. The texture depth should therefore be less than the maximum height of the binding material. Since fretting is defined as the loss of aggregate [3], conservatively a peak with a height which is at least double the height of a texture peak is considered to be fretting.

2) For similar reasons to the above, a broad peak is more likely to indicate road fretting. Therefore, a peak with at least double the width of the texture peak is considered to be fretting.

3) The minimum peak distance is used to prevent the over counting of the peaks in a short distance. It is set as the twice of the average peak distance.

4) Peak prominence is also used to ensure that a small peak is not mislabelled as fretting by judging the prominence value. It is also set as twice the average peak prominence.

The fretting detection algorithm proceeds by applying the above four rules for each peak $\lambda_{i}$. Only a peak which meets all of the above rules is considered as fretting. The output from this process is the detected number of peaks from one point laser. In this work, the zero-crossing algorithm as described in [40] for peak detection was adopted due to its ability to process time-series data quickly and its comparatively low computational power requirement. The effectiveness of the proposed fretting detection is demonstrated in Section V.

\section{Use of Multiple Point Laser}

The HARRIS2 system has an array of five point lasers (see Fig. 1), and this section describes how the proposed methodology combines the results, obtained using the above algorithm, from all five lasers. An approach could be to use the sensor fusion technique described in [41]. However, the fusion process at the data and feature level is highly complex due to the significant number of data points produced by the 
HARRIS2 system. For this reason, an empirical method has been developed which is based on the location of detected fretting by each point laser.

The location of each detected road fretting peak $y^{F}(t)$ is provided by the GPS system fitted on HARRIS2. Fretting is unlikely to extend completely across the road surface in the transverse direction, nor equally in the longitudinal direction. Thus, the shape of fretting can be considered to be irregular with respect to the road surface. Therefore, it is highly possible that an area of fretting maybe detected by more than one point laser. The method compares the location of the fretting associated peaks recorded by each of the five point lasers and the peaks which are within a given distance of each other are considered to belong to the same area of fretting.

\section{EXPERIMENTAL RESULTS AND DISCUSSIONS}

In this section, the performance of the above fretting detection methodology is demonstrated using the data set described in Section III C. The feasibility of the proposed concepts is verified by comparing the results with those obtained from a visual survey.

\section{A. Comparison With Vision Data}

The visual condition of the five surveyed roads was assessed using the Detailed Visual Inspection (DVI) method for fretting [3]. The DVI measures the fretting area (in square metres) for each $20 \mathrm{~m}$ length of a surveyed road (both the longitudinal and transverse directions are taken into account). Note that the algorithm described here for the measurement of fretting determines the fretting number (which is the number of detected peak as processed in Section IV), and is slightly different to the DVI measured fretting area. In order to allow for the comparison of the results between point laser and DVI data, the results from the point laser system have been evaluated every $20 \mathrm{~m}$. Accordingly, to allow for comparison with the DVI methodology, peaks identified by the current system within 20 metres of each other are considered to belong to the same area of fretting.

\section{B. Feasibility of the Proposed Peak Detection Method}

Firstly, the effectiveness of using each peak feature (Fig. 8) alone within the algorithm for fretting detection was investigated. Two measures were used to justify the performance of each individual peak feature, namely the Mean-Square-Error (MSE) and the correlation-coefficient (R).

The MSE value measures the error between the two datasets and is calculated as $M S E=\frac{1}{n} \sum_{i=1}^{n}\left|D V I_{i}-P_{i}\right|$, and the $\mathrm{R}$ value indicates the similarity between two datasets and is calculated as $R=\frac{\sum_{i=1}^{n} D V I_{i} P_{i}}{n \sigma_{D V I} \sigma P_{i}}$. Where $D V I_{i}$ is the normalised DVI reading for the $i^{\text {th }} 20 \mathrm{~m}$ length of road and $P_{i}$ represents the point laser data for the corresponding road segment. The results, considering all five roads together, are presented in Table III. As can be seen, among all features, peak height alone gives the most accurate result with an MSE of 0.93 and an $R$ value of -0.19 . In contrast, the peak distance showed the lowest accuracy as it has a very high MSE value of 15.02 and
TABLE III

Detection Performance by Using Each Single Feature

\begin{tabular}{|l|l|l|}
\hline & MSE & R \\
\hline Peak height & 0.93 & -0.19 \\
\hline Peak width & 1.99 & -0.19 \\
\hline Peak distance & 15.02 & -0.38 \\
\hline Peak prominence & 1.38 & -0.19 \\
\hline
\end{tabular}

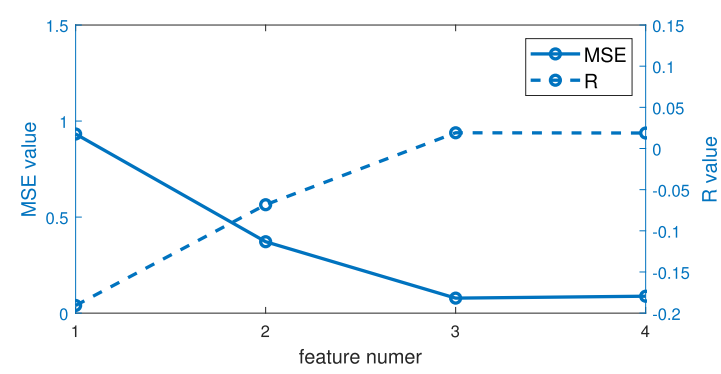

Fig. 9. Fretting detection result with different number of peak feature, MSE (solid line) and $\mathrm{R}$ (dashed line).

the lowest $\mathrm{R}$ value of -0.38 . This is unsurprising since both peak height and peak width are used to identify the peaks, whereas peak distance and peak prominence are used to prevent over counting. The high values in MSE and negative values in $\mathrm{R}$ indicate that a single peak feature cannot be used alone to detect fretting.

The performance of the system, when all of the features are used in combination, is calculated by adding each feature, one at a time, in the sequence of peak height, prominence, width and distance. The contribution of each feature to increasing the accuracy of fretting detection is shown in Fig. 9. As expected, the most accurate results are obtained when all four features are included, resulting in a smaller MSE value and a positive $\mathrm{R}$ value. Both peak width and prominence provide a large improvement in the accuracy. For the last feature, peak distance, the improvement is minimal when compared to the first three features and therefore a satisfactory performance could be achieved using only three features.

In order to demonstrate the feasibility of using the peak detection process for automated fretting detection, the example data shown in 6(c) has been used. The results are shown in Fig. 10 with detected peaks in (a) and identified fretting in (b). As can be seen, the road surface is generally smooth, with a number of medium sized troughs of approximately $0.5 \mathrm{~mm}-2 \mathrm{~mm}$ in depth and some more pronounced troughs in the vicinity of $15-30 \mathrm{~m}$ and $45 \mathrm{~m}$. Troughs of more than $5 \mathrm{~mm}$ in depth can also be seen, such as those near 10 and $40 \mathrm{~m}$ respectively. All these areas are successfully detected by using the proposed methodology as can be seen by comparing Fig. 10(a) and (b). In comparison, small peaks due to varying road texture are removed by applying the feature vector $\lambda_{T}$. This indicates that the proposed automated detection can effectively remove the effect of road texture. It was noticed from a visual comparison of the data that some of the peaks identified by the automated system do not represent road fretting but are associated with other factors, for example manhole covers and speedbumps. This interference 

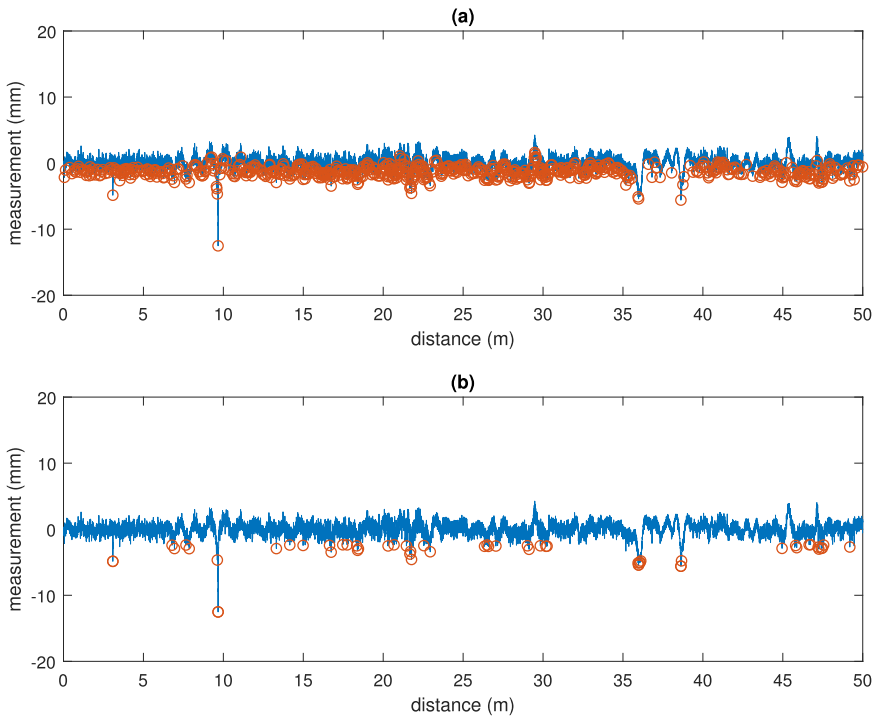

Fig. 10. An example of road fretting by proposed methodology, (a) all detected peaks and (b) extracted peaks based on the peak feature.

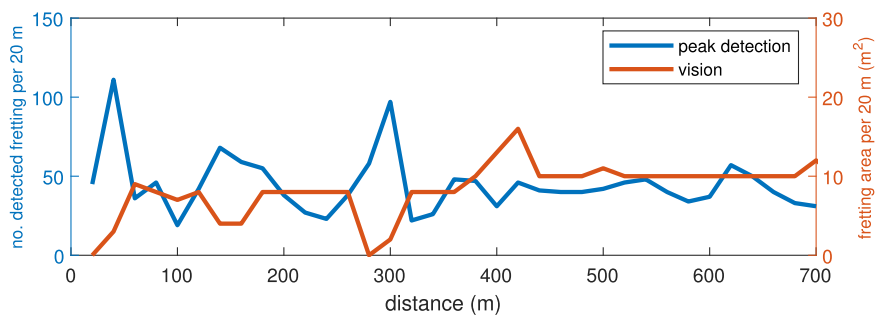

Fig. 11. Fretting detection of road one.

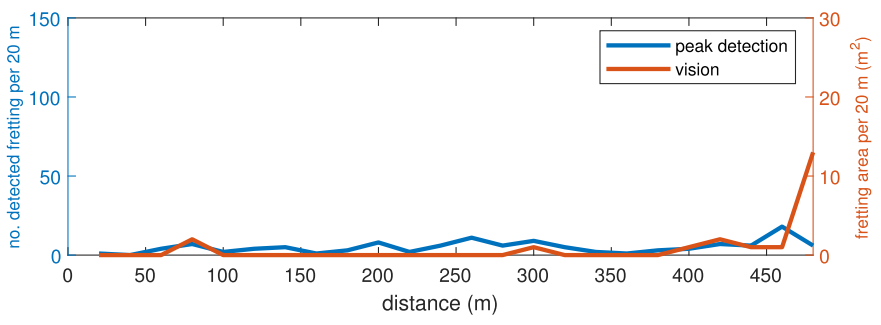

Fig. 12. Fretting detection of road two.

will undoubtedly affect the accuracy of detecting fretting, although it would only have a small impact on the system performance, since the road surface occupies a much greater area than such features.

\section{Results From Each Road}

The applicability of the proposed concepts was further explored by comparing the automated and DVI survey results for all five roads separately. The data from each point laser was segmented and fixed to a window length for the baseline correction of 20 metres to meet the corresponding standard of DVI measurement. Each road was processed individually.

The results from the point laser and DVI survey approaches are plotted in Figs 11-15 respectively. As the figures show, despite there being some differences between the two systems,

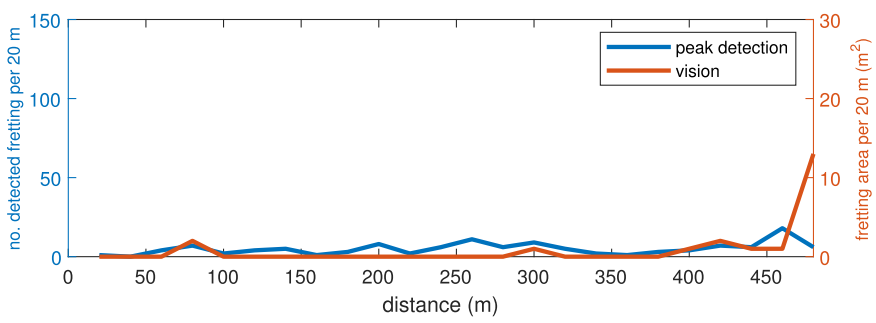

Fig. 13. Fretting detection of road three.

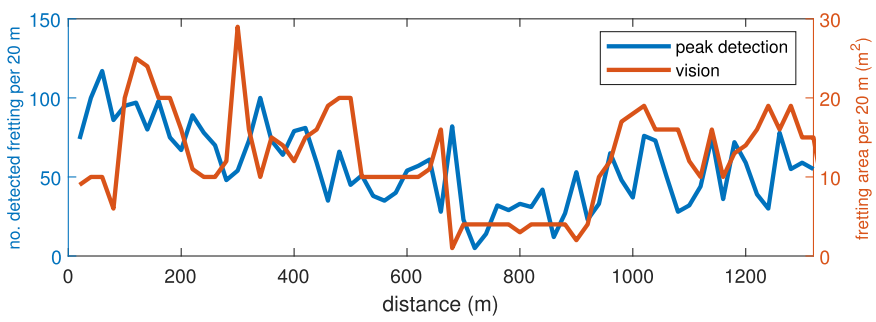

Fig. 14. Fretting detection of road four.

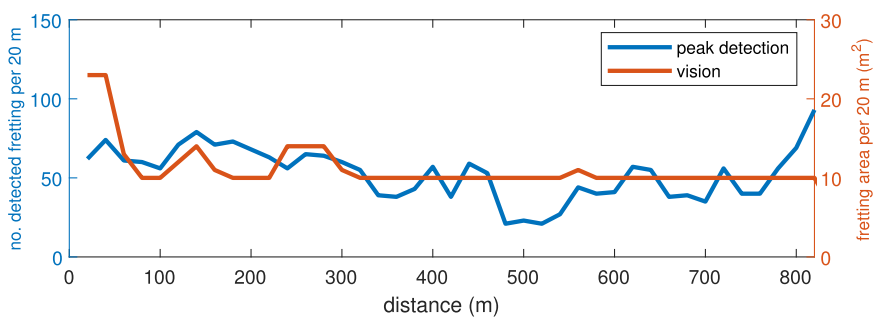

Fig. 15. Fretting detection of road five.

the detection of fretting is similar for the two systems. For example, both the automated and visual detection systems show greater amounts of fretting on roads one, four and five compared to roads two and three. Also, the automated system shows that road four has low amounts of fretting between 700-900 $\mathrm{m}$ and comparably high fretting numbers in other sections of the road. These results are similar to the visual data but with some differences. The reason for this is because the visually collected DVI data is in terms of the fretting area, whereas the point laser gives fretting numbers (or fretting intensity). For example, a small fretting area may contain a higher fretting number than a larger fretting area. Moreover, the detected fretting numbers in Figs 12,13 are much less than compared to those in Figs 11, 14 and 15. This indicates that the proposed methodology can easily distinguish the difference between a flat and an uneven surface. However, it can be seen that in a few instances there is a mismatch between the fretting computed by the automated system and that identified visually. This is particularly the case for road one. For example, at approximately $300 \mathrm{~m}$, the automated system computes a large amount of fretting and the visual survey suggests that the fretting is low. On investigation, it appears that the cause of this mismatch is due to a sudden change in the road texture which interrupts the calculation of the feature vector $\lambda_{T}$ used to identify the road texture. This results in an error in the peak detection process and a corresponding 


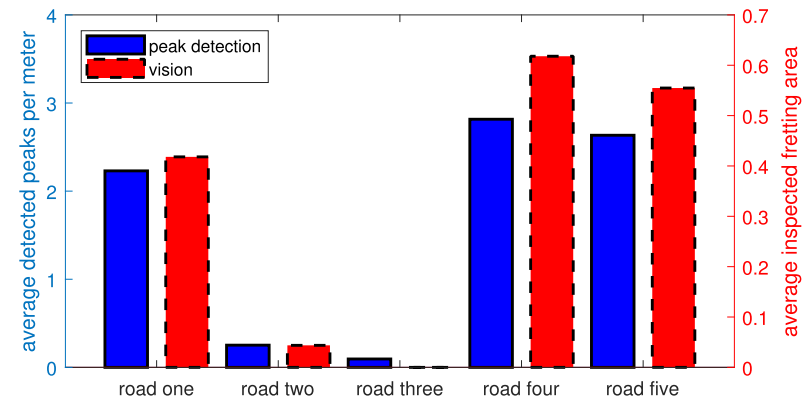

Fig. 16. Average detected fretting number and inspected fretting area of each road survey.

TABLE IV

Paired Difference Test Between Point Laser And DVI Data

\begin{tabular}{|l|l|l|l|l|l|l|}
\hline Road & $n$ & $\left(f_{a}-f_{n}\right) / n$ & $\begin{array}{l}\text { Standard } \\
\text { Deviation }\end{array}$ & $\begin{array}{l}\text { Standard } \\
\text { Error }\end{array}$ & $t$ & $t_{0.05}$ \\
\hline one & 35 & 0.17 & 0.92 & 0.16 & 1.10 & \pm 2.048 \\
\hline two & 24 & -0.04 & 0.20 & 0.42 & -1 & \pm 2.064 \\
\hline three & 21 & 0 & 4.48 & 0.98 & 0 & \pm 2.086 \\
\hline four & 65 & 0.11 & 0.98 & 0.12 & 0.88 & \pm 2.002 \\
\hline five & 41 & 0.15 & 0.69 & 0.11 & 0.136 & \pm 2.056 \\
\hline
\end{tabular}

misclassification of fretting. A potential solution could be to utilise an adaptive feature vector that adapts according to the change in road texture not just to distance along the road, for example training a classifier to recognise the difference between good and damaged road surface.

To further present the detection performance of the point laser system, the average fretting number per metre and the average inspected fretting area based on the whole road are shown in Fig. 16. The results from the proposed system show a high similarity to those provided by visual inspection. Road three is in better condition than road two despite both being considered to be in a good road condition. There are also some differences among the roads with higher amounts of fretting. Road four shows the highest average fretting compared to roads one and five and follows the same result as the vision data. These results indicate that the overall detection performance of the point laser system improves as the distance or road assessed increases.

For road maintenance management purposes road surface condition assessment, where is carried out, is usually categorised into levels of condition to facilitate maintenance decision making [37]. Data to such a level of detail is classified as Information Quality Level 3 (IQL 3) according to the World Bank's system for classifying data. Data at IQL 3 is suitable to support planning road management activities (i.e. long-term, strategic estimates of maintenance expenditure under budgetary scenarios) and programming management activities (i.e. the identification of sections of the local road network requiring treatment and the selection of the required treatments). Accordingly, the fretting data of each road were categorized into fretting levels of very low, low, moderate, high and very high amounts of road fretting as suggested by [37].
Further, the $20 \mathrm{~m}$ long sections once categorised, were compared on a road by road basis statistically using a Paired Difference Test [42]. The results of the statistical analysis are given in Table IV, where $n$ is the number of measurement, $f_{a}$ is the fretting area from the DVI data and $f_{n}$ is the fretting number from the newly proposed methodology. As can be seen, all standard deviation and standard error are generally small apart from road three which is due to the zero values in the DVI data. It may be seen that statistically, at the 95\% confidence level, there is no difference between the new methodology presented here and the manual approach in terms of categorising sections of all five roads according to the levels of fretting present. The proposed system can therefore be regarded as being suitable to collect road condition data to IQL 3 and may therefore be used for strategic planning and programming road management activities [37].

\section{CONCLUSION}

A robust, automated approach using a multi-point laser system for the rapid assessment of a measure of road condition known as fretting has been presented. The new low-complexity signal processing approach can accurately identify areas of fretting on the road surface from data obtained from a real road condition survey. A filtering process was outlined which removes noise associated with the sensor and the dynamic motions of the survey vehicle. Areas of fretting were successfully identified by removing parts of the signal due to the road texture using a peak feature vector. The experimental results indicated that the point laser system together with the associated proposed signal processing could provide sufficient performance for fretting detection. A statistical analysis demonstrated that the developed system is capable of classifying the amount of fretting occurring in short road sections as accurately as a detailed visual inspection. Accordingly, the proposed system can provide data to IQL 3 which is sufficient for the purposes of rapidly screening an entire road network to identify road sections requiring preventative maintenance and to determine the required treatments. Data collected to IQL 3 is also suitable for planning purposes, namely preparing strategic estimates of expenditure for road development and preservation under budgetary scenarios. However, further research would be necessary to the system for condition surveys to be carried out to IQL 1 and 2. This would allow the system to undertake a very detailed assessment for research purposes, advanced design and detailed diagnosis of short sections of road (typically $<5 \mathrm{~m}$ ). Such improvements to the system could be made by using the inertial information from the inertial measurement unit to allow for the vehicle's dynamic motion and thus negate the need for the filtering approach suggested here. Furthermore, an adaptive feature vector that can be modified according to the change in road texture, could be developed to allow for improved accuracy where there are abrupt changes in road texture. Whilst the research has focused on the assessment of fretting it is recognised that the measurement of other parameters such as cracking, roughness and skid resistance are required for a comprehensive assessment of the functional performance of rural roads. 


\section{ACKNOWLEDGMENT}

The practical contribution of the Department of Civil Engineering at the University of Birmingham, UK is appreciated. We also gratefully acknowledge the support from Highways Surveyors Ltd and the Transport Research Laboratory (TRL) Ltd which provided road condition data.

\section{REFERENCES}

[1] E. Ivanova and J. Masarova, "Importance of road infrastructure in the economic development and competitiveness," Econ. Manage., vol. 18, no. 2, pp. 263-274, 2013.

[2] S. Mathavan, K. Kamal, and M. Rahman, "A review of three-dimensional imaging technologies for pavement distress detection and measurements," IEEE Trans. Intell. Transp. Syst., vol. 16, no. 5, pp. 2353-2362, Oct. 2015.

[3] Chapter 8: Detailed visual inspection (DVI). Accessed: Nov. 29, 2019. [Online]. Available: http://www.ukroadsliaisongroup. org/en/utilities/documentsummary.cfm?docid=6C39F16DB012-43BB-956247C5DFCD10F6

[4] H. Guan, J. Li, Y. Yu, M. Chapman, and C. Wang, "Automated road information extraction from mobile laser scanning data," IEEE Trans. Intell. Transp. Syst., vol. 16, no. 1, pp. 194-205, Feb. 2015

[5] Road Conditions in England: 2016. Accessed: Nov. 29, 2019. [Online]. Available: https://www.gov.uk/government/statistics/road-conditions-inengland-2016

[6] A. Wright et al., "New methods for network level automated assessment of surface condition in the UK," in Proc. 7th Symp. Pavement Surf. Characteristics (SURF), 2012.

[7] J. Laurent, J. F. Hébert, D. Lefebvre, and Y. Savard, "Using 3D laser profiling sensors for the automated measurement of road surface conditions," in Proc. 7th RILEM Int. Conf. Cracking Pavements. Dordrecht, The Netherlands: Springer, 2012, pp. 157-167.

[8] S. McRobbie, A. Wright, J. Iaquinta, P. Scott, C. Christie, and D. James, "Developing new methods for the automatic measurement of raveling at traffic-speed," in Proc. 11th Int. Conf. Asphalt Pavements Nagoya, Japan, 2010, p. 10.

[9] M. Aki et al., "Road surface recognition using laser radar for automatic platooning," IEEE Trans. Intell. Transp. Syst., vol. 17, no. 10, pp. 2800-2810, Oct. 2016.

[10] H. Viner, P. Abbott, A. Dunford, N. Dhillon, L. Parsley, and C. Read, "Surface texture measurement on local roads," Project Rep. PPR148, TRL, Crowthorne, U.K., 2006.

[11] N. Ryden and C. B. Park, "Fast simulated annealing inversion of surface waves on pavement using phase-velocity spectra," Geophysics, vol. 71, no. 4, pp. R49-R58, 2006.

[12] Y. Taniguchi, K. Nishii, and H. Hisamatsu, "Evaluation of a bicyclemounted ultrasonic distance sensor for monitoring road surface condition," in Proc. 7th Int. Conf. Comput. Intell., Commun. Syst. Netw., Jun. 2015, pp. 31-34.

[13] S. E. Paje, M. Bueno, F. Terán, U. Viñuela, and J. Luong, "Assessment of asphalt concrete acoustic performance in urban streets," J. Acoust. Soc. Amer, vol. 123, no. 3, pp. 1439-1445, 2008.

[14] S. Viollon and C. Lavandier, "Multidimensional assessment of the acoustic quality of urban environments," in Proc. 29th Int. Congr. Exhib. Noise Control Eng., vol. 4, 2000, pp. 2279-2284.

[15] S. R. Pennock and C. H. J. Jenks, "Road surface and pavement condition assessment by high frequency GPR diffraction," in Proc. 15th Int. Conf. Ground Penetrating Radar, Jun./Jul. 2014, pp. 881-886.

[16] M. Graczyk, L. Krysiński, Ł. Topczewski, and J. Sudyka, "The use of three-dimensional analysis of GPR data in evaluation of operational safety of airfield pavements," Transp. Res. Procedia, vol. 14, pp. 3704-3712, Jan. 2016.

[17] M.-C. Amann, T. M. Bosch, M. Lescure, R. A. Myllylae, and M. Rioux, "Laser ranging: A critical review of unusual techniques for distance measurement," Opt. Eng., vol. 40, no. 1, pp. 10-20, 2001.

[18] M. Cheng, H. Zhang, C. Wang, and J. Li, "Extraction and classification of road markings using mobile laser scanning point clouds," IEEE J. Sel. Topics Appl. Earth Observ. Remote Sens., vol. 10, no. 3, pp. 1182-1196, Mar. 2017.
[19] L. Zhang, F. Yang, Y. D. Zhang, and Y. J. Zhu, "Road crack detection using deep convolutional neural network," in Proc. IEEE Int. Conf. Image Process. (ICIP), Sep. 2016, pp. 3708-3712.

[20] T. Yamaguchi, S. Nakamura, R. Saegusa, and S. Hashimoto, "Imagebased crack detection for real concrete surfaces," IEEJ Trans. Elect. Electron. Eng., vol. 3, no. 1, pp. 128-135, Jan. 2008.

[21] T. Nishikawa, J. Yoshida, T. Sugiyama, and Y. Fujino, "Concrete crack detection by multiple sequential image filtering," Comput.-Aided Civil Infrastruct. Eng., vol. 27, no. 1, pp. 29-47, 2012.

[22] J. Han, D. Kim, M. Lee, and M. Sunwoo, "Enhanced road boundary and obstacle detection using a downward-looking LIDAR sensor," IEEE Trans. Veh. Technol., vol. 61, no. 3, pp. 971-985, Mar. 2012.

[23] S. Chambon and J.-M. Moliard, "Automatic road pavement assessment with image processing: Review and comparison," Int. J. Geophys., vol. 2011, Jun. 2011, Art. no. 989354.

[24] C. Yang, Z. He, and W. Yu, "Comparison of public peak detection algorithms for MALDI mass spectrometry data analysis," BMC Bioinf., vol. 10, no. 1, 2009, Art. no. 4.

[25] S.-W. Lee and K. Mase, "Activity and location recognition using wearable sensors," IEEE Pervasive Comput., vol. 1, no. 3, pp. 24-32, Jul./Sep. 2002.

[26] S. Hemminki, P. Nurmi, and S. Tarkoma, "Accelerometer-based transportation mode detection on smartphones," in Proc. 11th ACM Conf. Embedded Netw. Sensor Syst., Nov. 2013, Art. no. 13.

[27] S. AminiKhanghahi, T. Wang, and D. J. Cook, "Real-time change point detection with application to smart home time series data," IEEE Trans. Knowl. Data Eng., vol. 31, no. 5, pp. 1010-1023, May 2019.

[28] J. Reeves, J. Chen, X. L. Wang, R. Lund, and Q. Q. Lu, "A review and comparison of changepoint detection techniques for climate data," J. Appl. Meteorol. Climatol., vol. 46, no. 6, pp. 900-915, 2007.

[29] R. Rawassizadeh, E. Momeni, C. Dobbins, J. Gharibshah, and M. Pazzani, "Scalable daily human behavioral pattern mining from multivariate temporal data," IEEE Trans. Knowl. Data Eng., vol. 28, no. 11, pp. 3098-3112, Nov. 2016.

[30] M. F. R. Chowdhury, S.-A. Selouani, and D. O'Shaughnessy, "Bayesian on-line spectral change point detection: A soft computing approach for on-line ASR," Int. J. Speech Technol., vol. 15, no. 1, pp. 5-23, 2012.

[31] M. Han, L. T. Vinh, Y.-K. Lee, and S. Lee, "Comprehensive context recognizer based on multimodal sensors in a smartphone," Sensors, vol. 12, no. 9, pp. 12588-12605, 2012.

[32] S. Hido, T. Idé, H. Kashima, H. Kubo, and H. Matsuzawa, "Unsupervised change analysis using supervised learning," in Proc. Pacific-Asia Conf. Knowl. Discovery Data Mining. Berlin, Germany: Springer, 2008, pp. $148-159$.

[33] Z. Harchaoui, F. Vallet, A. Lung-Yut-Fong, and O. Cappe, "A regularized kernel-based approach to unsupervised audio segmentation," in Proc. IEEE Int. Conf. Acoust., Speech Signal Process. (ICASSP), Apr. 2009, pp. $1665-1668$.

[34] Proficura 2D. Accessed: Nov. 29, 2019. [Online]. Available: http:// www.limab.com/products/proficura-2d-2/

[35] Laser Crack Measurement System (LCMS). [Online]. Available: http://www.pavemetrics.com/applications/road-inspection/lasercrackmeasurement-system/\#1439492605807-40e2dfd3-f083

[36] L. L. Scharf and C. Demeure, Statistical Signal Processing: Detection, Estimation, and Time Series Analysis, vol. 63. Reading, MA, USA: Addison-Wesley, 1991.

[37] R. Robinson and B. Thagesen, Road Engineering for Development. Boca Raton, FL, USA: CRC Press, 2004.

[38] S. E. Said and D. A. Dickey, "Testing for unit roots in autoregressivemoving average models of unknown order," Biometrika, vol. 71, no. 3, pp. 599-607, 1984.

[39] A. Savitzky and M. J. E. Golay, "Smoothing and differentiation of data by simplified least squares procedures," Anal. Chem., vol. 36, no. 8 , pp. 1627-1639, 1964.

[40] Peak Finding and Measurement. Accessed: Nov. 29, 2019. [Online]. Available: https://terpconnect.umd.edu/ toh/spectrum/ PeakFindingandMeasurement.htm

[41] W. Elmenreich, An Introduction to Sensor Fusion, vol. 502. Vienna, Austria: Vienna Univ. of Technology, 2002.

[42] H. A. David, The Method of Paired Comparisons, vol. 12. Vienna, Austria: Vienna Univ. of Technology, Department of Computer Engineering, 1963. 


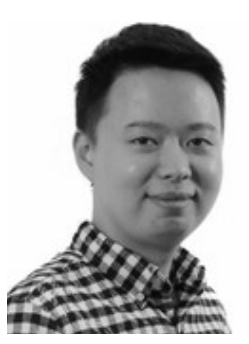

Wenda Li received the M.Eng. and Ph.D. degrees from the University of Bristol, in 2013 and 2017, respectively. He is currently an Honorary Research Fellow with the Department of Civil Engineering, University of Birmingham. His research focuses on the signal processing for radar-based applications, including passive WiFi radar and penetrate ground radar. He is also interested in sensory signal analysis for autonomous vehicle and automatic road network assessment.

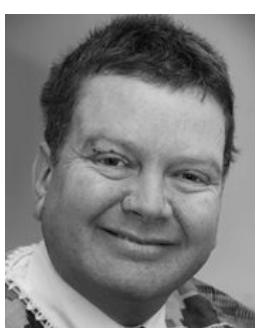

Michael Burrow received the M.A. degree in engineering from the University of Cambridge and the $\mathrm{Ph} . \mathrm{D}$. degree in road asset management from the University of Birmingham. He is currently a Senior Lecturer with the School of Engineering, University of Birmingham. His research and teaching is associated with infrastructure asset management, he has supervised over $40 \mathrm{Ph} . \mathrm{D}$. students and has published 90 peer reviewed articles in the subject area. He has forged close links with industry and academia through his research and has a result worked with partners from over 23 countries.

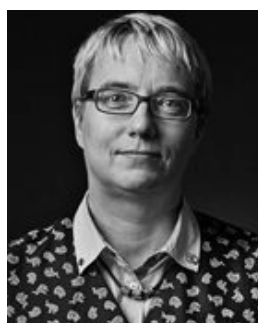

Nicole Metje is currently a Professor of infrastructure monitoring with the University of Birmingham. She is the Deputy Director of sensors of the UKCRIC National Buried Infrastructure Facility. She leads the Geophysics Stream of Birmingham's Quantum Technology Hub. Her research focusses on detection of buried infrastructure and the development and use of sensing technologies to see through the ground and/or assess the condition of infrastructure assets. She is an Active Member of the steering committee for BSI's PAS128 Specification for underground utility detection, verification and location, and for the update of ASCE38.

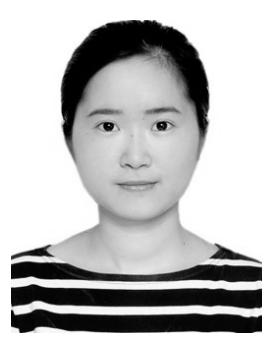

Yueyue Tao received the B.E. degree in transportation engineering from Nanjing Agricultural University, Nanjing, China, in 2015 , the M.S. degree in road management and engineering from the University of Birmingham, Birmingham, U.K., in 2017, where she is currently pursuing the Ph.D. degree in civil engineering. Her current research topic is automatic detection and classification of defects on road pavement. Her research interests include road management, computer vision, and signal processing applied on the road assessment.

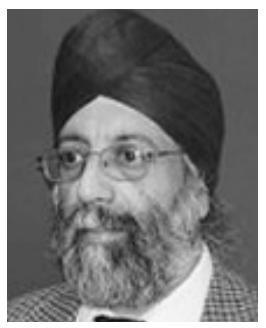

Gurmel Ghataora received the Ph.D. degree. He is currently a Geotechnical Engineer. He has extensive experience in site investigation, materials testing (both laboratory and field), ground improvement, use of out-of-specification materials in construction, and improvement and design of both rural roads and railway track foundations. He has supervised 61 research students, and he has published chapters in a number of books and has over 140 peer reviewed journals and conference papers. He has a substantial number of these are on materials for rural roads and related topics. He has been a member of the TRB committees AFS90 Chemical and Mechanical Stabilization and AR060-Railway Maintenance. 